\title{
Modeling and Simulation of the Silicon Micro-machined Gyroscope Mode-Coupling System
}

\author{
Qirui $\mathrm{Li}^{1, \mathrm{a}}$, Weihong $\mathrm{Jin}^{1, \mathrm{~b}}$, Haimei $\mathrm{Xu}^{1, \mathrm{c}}$ \\ ${ }^{1}$ Chongqing Communication Institute, Chongqing 400035, China \\ a18680950214@163.com, b168106572@qq.com, cxuhaimei@uestc.edu,cn
}

Keywords: Silicon Micro-machined Gyroscope, Mode-Coupling, Modeling, Simulation

\begin{abstract}
This paper focuses on the mode-coupling characteristics of gyroscope, and tries to rebuild a more perfect system model of silicon micro-machined gyroscope. It analyses the factors causing the coupling motions between drive-mode and detect-mode of gyroscope system. The mathematical model is deducted out based on the dynamic model and dynamic equations of gyroscope system. And the simulation resulted by MATLAB is in good agreement with the experiment results, which verifies the correctness of the mathematical model of silicon micro-machined gyroscope mode-coupling system.
\end{abstract}

\section{Introduction}

Silicon micro-machined gyroscope can be widely used in military and civilian fields for its smaller size, lower power consumption and lower price [1]. To improve its controlling and measuring accuracy has always been one of the main research contents in silicon micro-machined gyroscope's development.

System model is the foundation of system controlling and measuring, and hence the accuracy of gyroscope's mathematical model has a great influence on the performance of silicon micro-machined gyroscope system. Building a more accurate mathematical model of silicon micro-machined gyroscope is therefore a way to improve gyroscope system's controlling and measuring accuracy.

The drive-mode and detect-mode of silicon micro-machined gyroscope are coupled [2]. However, they are usually considered as two full decoupling modes, while the mathematical model of gyroscope is equivalent to a second order system model approximately, regardless of non-ideal factors such as mode-coupling. To improve the accuracy and adequacy of gyroscope system model, this paper tries to rebuild a more accurate mathematical model of silicon micro-machined gyroscope concerning the factors of drive-mode and detect-mode coupling, which can provide forceful support for the future studies on the silicon micro-machined gyroscope mode-coupling system.

\section{Idealized Mathematical Model of Silicon Micro-machined Gyroscope System}
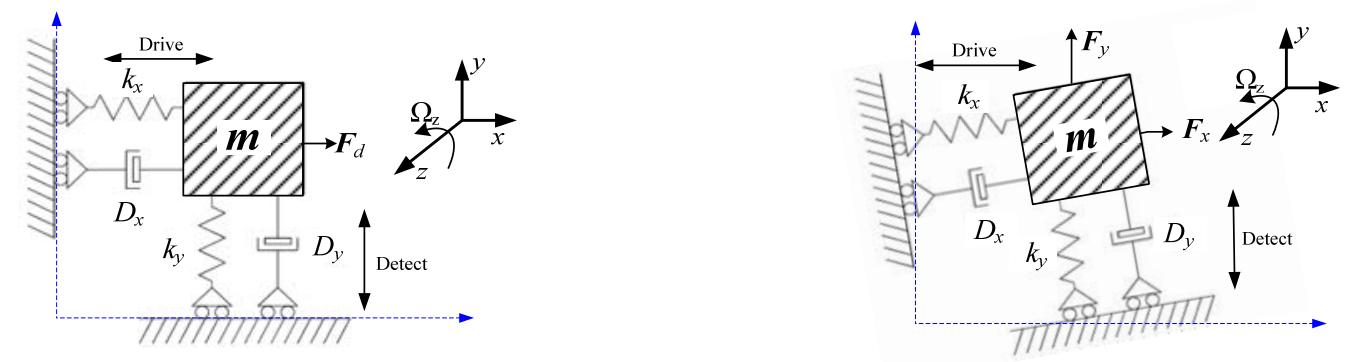

a. Idealized equivalent dynamic model $\quad$ b. Non-idealized equivalent dynamic model

Fig. 1 Equivalent Dynamic Model

Equivalent Dynamic Model. Ideally, silicon micro-machined gyroscope system is equivalent to a second order dynamic system as shown in Fig.1.a, where $x$-direction represents drive-mode direction, $y$-direction represents detect-mode direction, and z-direction represents gyroscope's angular velocity 
measuring direction. $D_{x}, D_{y}$ and $k_{x}, k_{y}$ indicate the damping coefficients and elasticity coefficients in drive-mode direction and detect-mode direction, respectively. And $\Omega_{\mathrm{z}}$ indicates the angular velocity in $z$-direction. In the ideal case, the moving mode in $x$-direction of the mass $m$ and its moving mode in $y$-direction are considered as full decoupled, which means that either one of the movements in drive-mode or detect-mode direction won't affect the movement in another direction.

Equivalent Dynamic Equations. Suppose that a driving force $F_{d}$ is imposed on the mass $m$ in the $x$-direction, and the equivalent dynamic system (gyroscope) rotates around $z$-direction. In the reference $x y z$-axes system, the mass $m$ is subjected by Driving Force, Damping Force, Elasticity Force and Coriolis force in the $x y$-plane. Based on the above force analysis and Newton's Second Law, the dynamic equations are derived out, and the idealized mathematical model of silicon micro-machined gyroscope system is often written as the following simultaneous differential equations[3]:

$$
\left\{\begin{array}{c}
m \ddot{x}+D_{x} \dot{x}+k_{x} x=F_{d} \\
m \ddot{y}+D_{y} \dot{y}+k_{y} y=-2 m \Omega_{z} \dot{x}
\end{array}\right.
$$

\section{Analysis of Silicon Micro-machined Gyroscope's Mode-Coupling Characteristics}

Frequency Domain Analysis. Accoding to Equation(1), supposing to impose driving force $F_{d x}$ in the drive-mode direction and assuming $\Omega_{\mathrm{z}}=0$, the system transfer function of gyroscope's drive-mode is derived by Laplace Transformation as Equantion(2). In the same way, the system transfer function of the detect-mode is written as Equantion(3). Plug the gyroscope parameters into the transfer functions, the frequency response curves are obtained by MATLAB as shown in Fig. 2 .

$$
\begin{aligned}
& G_{x}(s)=\frac{X(s)}{F_{d x}(s)}=\frac{1}{m s^{2}+D_{x} s+k_{x}} \\
& G_{y}(s)=\frac{X(s)}{F_{d y}(s)}=\frac{1}{m s^{2}+D_{y} s+k_{y}}
\end{aligned}
$$
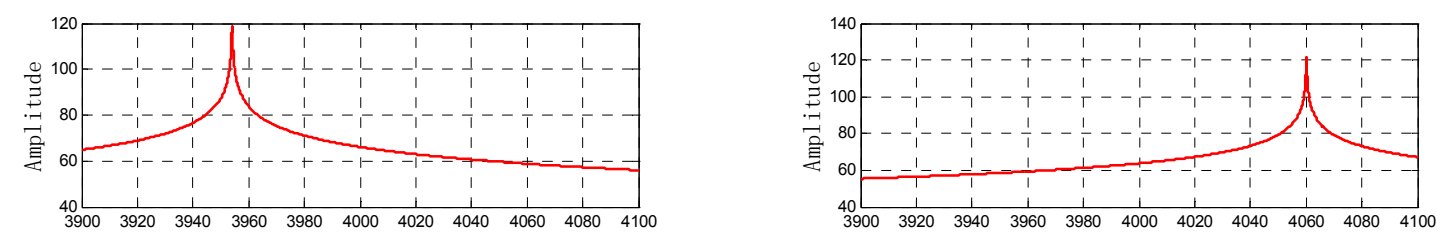

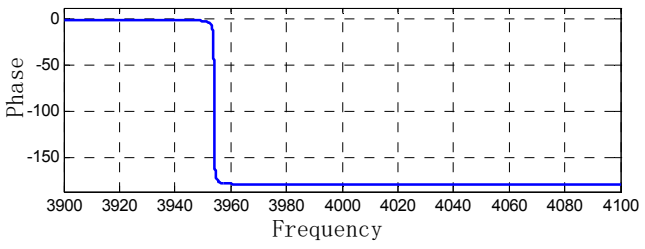

(a) Frequency response curves of $G_{x}(s)$

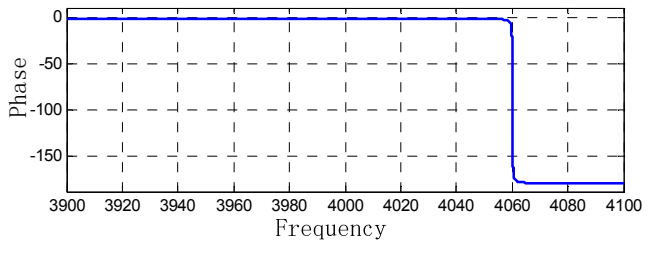

(b) Frequency response curves of $G_{y}(s)$

Fig.2 Frequency Response Curves of $G_{x}(s)$ and $G_{y}(s)$ 


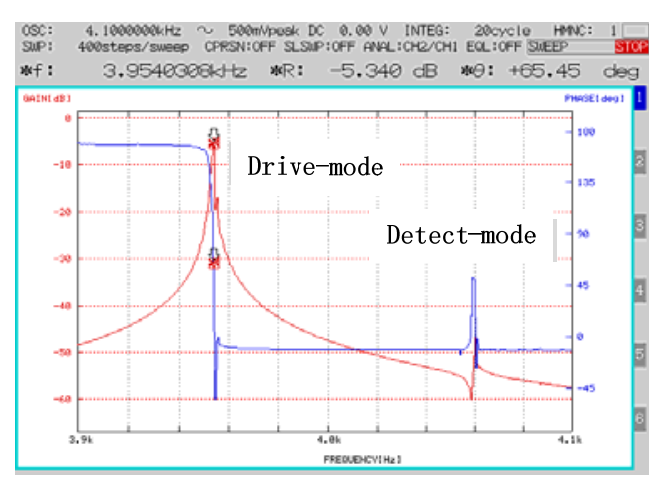

(a) Measurement result of drive-mode

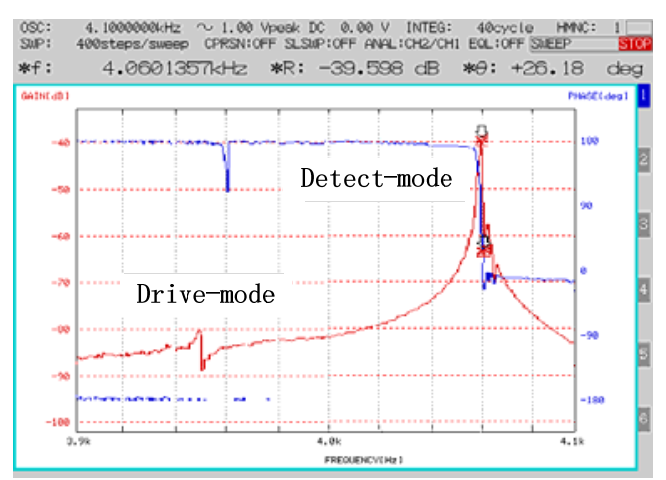

(b) Measurement result of detect-mode

Fig.3 Frequency Response Measurement Results

Frequency Response Measurement. However, the results of the frequency response measuring experiment for a certain real silicon micro-machined gyroscope system is shown as Fig.3. It indicates that the frequecy response cuves of drive-mode has a step change at the resonance frequency of detect-mode, while the cuves of detect-mode also has a step change at the resonance frequency of drive-mode, which implies that the drive-mode and detect-mode of the gyroscope system are coupling.

Mode-Coupling Characteristic Analysis. Silicon micro-machined gyroscope is one kind of vibrational sensors. It is based on the vibrational motions of gyroscope's silicon micro-machined vibrational structure to sense the angular velocity. Ideally, the vibrational motions of gyroscope's drive-mode and detect-mode are completly orthogonal, while the vibrational motions are coupling accuratly[2]. There are three main sides of non-ideal factors causing the coupling motions between drive-mode and detect-mode: (1) Structural asymmetry. Becaue of mismachining tolerance and material defect, gyroscope's silicon micro-machined vibrational structure is asymmetric, which arouses the asymmetry of rotational inertia and non-idealized movements. (2)Unbalanced driving force. The driving forces are imposed on the vibrational structure through driving electrodes. Due to sructural and cricuit errors, the driving forces imposed on the structure are unbalanced, which causes non-idealized dynamic coupling movements. (3)Principium factor. In the design process of silicon micro-machined gyroscope system, some approximate deposals in principle lead to the neglect of the coupling characteristics of the system itself, including the coupling motions between drive-mode and detect-mode.

\section{Mathematical Model Deduction for the Mode-Coupling System}

Considering the mode-coupling characteristics of the silicon micro-machined gyroscope system, its equivalent dynamic model is shown as Fig.1.b, which identifies the motions in drive-mode direction and detect-mode direction are non-orthogonal. Assuming the resultant forces in the $x$-direction and $y$-direction are respectively $F_{x}, F_{y}$, Equation (4) and (5) are written as following, based on Newton's Second Law. In the equations, $D_{x x}$ and $k_{x x}$ are the components of drive-mode's damping and elasticity coefficients in $x$-axis, $\Delta D_{y}$ and $\Delta k_{y}$ are the components of detect-mode's damping and elasticity coefficients in $x$-axis, $D_{y y}$ and $k_{y y}$ are the components of detect-mode's damping and elasticity coefficients in $y$-axis, $\Delta D_{x}$ and $\Delta k_{x}$ are the components of drive-mode's damping and elasticity coefficients in $y$-axis, $a_{x}$ and $a_{y}$ indicate the absolute accelerations of the mass $m$ in $x$-direction and $y$-direction.

$$
\begin{aligned}
& m a_{x}=F_{x}-k_{x x} x-D_{x x} \dot{x}-\Delta k_{y} y-\Delta D_{y} \dot{y} \\
& m a_{y}=F_{y}-k_{y y} y-D_{y y} \dot{y}-\Delta k_{x} x-\Delta D_{x} \dot{x}
\end{aligned}
$$


Refer to the deduction process of Coriolis acceleration, the absolute acceleration $\boldsymbol{a}$ of the mass $m$ is shown as Equation (6) [4]. $\boldsymbol{r}$ is the absolute vector of the mass $m$ in the inertial reference frame, $\Omega$ indicates the angular velocity of senor system.

$$
\boldsymbol{a}=\left[\begin{array}{lll}
a_{x} & a_{y} & a_{z}
\end{array}\right]^{T}=\frac{\partial^{2} \boldsymbol{r}}{\partial t^{2}}+2 \Omega \times \frac{\partial \boldsymbol{r}}{\partial t}+\frac{\partial \Omega}{\partial t} \times \boldsymbol{r}+\Omega \times \Omega \times \boldsymbol{r}
$$

Given $\boldsymbol{r}=\left[\begin{array}{lll}x & y & z\end{array}\right]^{T}, \Omega=\left[\begin{array}{lll}\Omega_{x} & \Omega_{y} & \Omega_{z}\end{array}\right]^{T}$ then Equation (6) is rewritten as following:

$$
\begin{aligned}
\boldsymbol{a} & =\left[\begin{array}{l}
a_{x} \\
a_{y} \\
a_{z}
\end{array}\right]=\left[\begin{array}{c}
\ddot{x} \\
\ddot{y} \\
\ddot{z}
\end{array}\right]+2\left[\begin{array}{l}
\Omega_{x} \\
\Omega_{y} \\
\Omega_{z}
\end{array}\right] \times\left[\begin{array}{c}
\dot{x} \\
\dot{y} \\
\dot{z}
\end{array}\right]+\left[\begin{array}{l}
\dot{\Omega}_{x} \\
\dot{\Omega}_{y} \\
\dot{\Omega_{z}}
\end{array}\right] \times\left[\begin{array}{l}
x \\
y \\
z
\end{array}\right]+\left[\begin{array}{l}
\Omega_{x} \\
\Omega_{y} \\
\Omega_{z}
\end{array}\right] \times\left[\begin{array}{l}
\Omega_{x} \\
\Omega_{y} \\
\Omega_{z}
\end{array}\right] \times\left[\begin{array}{c}
x \\
y \\
z
\end{array}\right] \\
& =\left[\begin{array}{c}
\ddot{x} \\
\ddot{y} \\
\ddot{z}
\end{array}\right]+\left[\begin{array}{ccc}
0 & -2 \Omega_{z} & 2 \Omega_{y} \\
2 \Omega_{z} & 0 & -2 \Omega_{x} \\
-2 \Omega_{y} & 2 \Omega_{x} & 0
\end{array}\right]\left[\begin{array}{l}
\dot{x} \\
\dot{y} \\
\dot{z}
\end{array}\right]+\left[\begin{array}{ccc}
-\left(\Omega_{y}^{2}+\Omega_{z}^{2}\right) & \Omega_{x} \Omega_{y}-\dot{\Omega}_{z} & \Omega_{x} \Omega_{z}+\dot{\Omega}_{y} \\
\Omega_{x} \Omega_{y}+\dot{\Omega}_{z} & -\left(\Omega_{x}^{2}+\Omega_{z}^{2}\right) & \Omega_{y} \Omega_{z}-\dot{\Omega}_{x} \\
\Omega_{x} \Omega_{z}-\dot{\Omega}_{y} & \Omega_{y} \Omega_{z}+\dot{\Omega}_{x} & -\left(\Omega_{x}^{2}+\Omega_{y}^{2}\right)
\end{array}\right]\left[\begin{array}{l}
x \\
y \\
z
\end{array}\right]
\end{aligned}
$$

So the components in $\mathrm{x}$-axis and $\mathrm{y}$-axis of the absolute acceleration $\boldsymbol{a}$ are written as:

$$
\begin{aligned}
& a_{x}=\ddot{x}-2 \Omega_{z} \dot{y}+2 \Omega_{y} \dot{z}-\left(\Omega_{y}^{2}+\Omega_{z}^{2}\right) x+\left(\Omega_{x} \Omega_{y}-\dot{\Omega}_{z}\right) y+\left(\Omega_{x} \Omega_{z}+\dot{\Omega}_{y}\right) z \\
& a_{y}=\ddot{y}+2 \Omega_{z} \dot{x}-2 \Omega_{x} \dot{z}+\left(\Omega_{x} \Omega_{y}+\dot{\Omega}_{z}\right) x-\left(\Omega_{y}^{2}+\Omega_{z}^{2}\right) y+\left(\Omega_{y} \Omega_{z}-\dot{\Omega}_{y}\right) z
\end{aligned}
$$

The motion along $z$-axis is not taken into account by the equivalent dynamic model, so it's considered that:

$Z=\dot{Z}=0$

Take Equation (8)(9)(10) into Equation (4)(5), then they convert to:

$$
\begin{aligned}
& m \ddot{x}+D_{x x} \dot{x}+\left(k_{x x}-m \Omega_{y}^{2}-m \Omega_{z}^{2}\right) x=F_{x}-\left(-2 m \Omega_{z}+\Delta D_{y}\right) \dot{y}-\left(m \Omega_{x} \Omega_{y}-m \dot{\Omega}_{z}+\Delta k_{y}\right) y \\
& m \ddot{y}+D_{y y} \dot{y}+\left(k_{y y}-m \Omega_{x}^{2}-m \Omega_{z}^{2}\right) y=F_{y}-\left(2 m \Omega_{z}+\Delta D_{x}\right) \dot{x}-\left(m \Omega_{x} \Omega_{y}+m \dot{\Omega}_{z}+\Delta k_{x}\right) x
\end{aligned}
$$

Combining Equation (11) and (12), the matrix equation, which presents the mathematical model of silicon micro-machined gyroscope mode-coupling system, is obtained as following:

$$
\begin{aligned}
& {\left[\begin{array}{cc}
m & 0 \\
0 & m
\end{array}\right]\left[\begin{array}{c}
\ddot{x} \\
\ddot{y}
\end{array}\right]+\left[\begin{array}{cc}
D_{x x} & \Delta D_{y} \\
\Delta D_{x} & D_{y y}
\end{array}\right]\left[\begin{array}{c}
\dot{x} \\
\dot{y}
\end{array}\right]+\left[\begin{array}{cc}
k_{x x}-m \Omega_{y}^{2}-m \Omega_{z}^{2} & m \Omega_{x} \Omega_{y}-m \dot{\Omega}_{z}+\Delta k_{y} \\
m \Omega_{x} \Omega_{y}+m \dot{\Omega}_{z}+\Delta k_{x} & k_{y y}-m \Omega_{x}^{2}-m \Omega_{z}^{2}
\end{array}\right]\left[\begin{array}{c}
x \\
y
\end{array}\right]} \\
& =\left[\begin{array}{cc}
0 & 2 m \Omega_{z} \\
-2 m \Omega_{z} & 0
\end{array}\right]\left[\begin{array}{c}
\dot{x} \\
\dot{y}
\end{array}\right]+\left[\begin{array}{c}
F_{x} \\
F_{y}
\end{array}\right]
\end{aligned}
$$

Assuming the above equation is equal to:

$$
\left[\begin{array}{cc}
m & 0 \\
0 & m
\end{array}\right]\left[\begin{array}{c}
\ddot{x} \\
\ddot{y}
\end{array}\right]+\left[\begin{array}{ll}
D_{11} & D_{12} \\
D_{21} & D_{22}
\end{array}\right]\left[\begin{array}{c}
\dot{x} \\
\dot{y}
\end{array}\right]+\left[\begin{array}{ll}
k_{11} & k_{12} \\
k_{21} & k_{22}
\end{array}\right]\left[\begin{array}{l}
x \\
y
\end{array}\right]=\left[\begin{array}{cc}
0 & 2 m \Omega_{z} \\
-2 m \Omega_{z} & 0
\end{array}\right]\left[\begin{array}{c}
\dot{x} \\
\dot{y}
\end{array}\right]+\left[\begin{array}{c}
F_{x} \\
F_{y}
\end{array}\right]
$$

Then, the equations are derived by Laplace Transformation as following:

$$
\begin{aligned}
& X(s)=\frac{\left(2 m \Omega_{z}-D_{12}\right) s-k_{12}}{m s^{2}+D_{11} s+k_{11}} Y(s)+\frac{1}{m s^{2}+D_{11} s+k_{11}} F_{x}(s)=G_{x y}(s) Y(s)+G_{F_{x}}(s) F_{x}(s) \\
& Y(s)=\frac{\left(-2 m \Omega_{z}-D_{21}\right) s-k_{21}}{m s^{2}+D_{22} s+k_{22}} X(s)+\frac{1}{m s^{2}+D_{22} s+k_{22}} F_{y}(s)=G_{y x}(s) Y(s)+G_{F_{y}}(s) F_{y}(s)
\end{aligned}
$$


According to Equation (15) and (16), the controlling structure diagram of silicon micro-machined gyroscope mode-coupling system is shown as Fig.4.

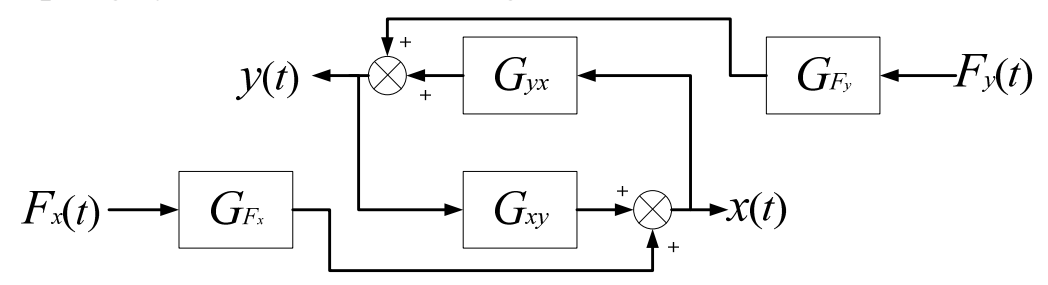

Fig.4 Controlling Structure Diagram of Mode-Coupling System

Therefore, depending on the mathematical model and controlling structure diagram, it is represented that silicon micro-machined gyroscope system is a multiple input multiple output (MIMO) system.

\section{Simulating Verification by MATLAB}

Based on Equation (15) and (16), the equations are obtained as following:

$$
\begin{aligned}
& X(s)=\frac{G_{F_{x}}(s)}{1-G_{x y}(s) \cdot G_{y x}(s)} F_{x}(s)+\frac{G_{x y}(s) \cdot G_{F_{y}}(s)}{1-G_{x y}(s) \cdot G_{y x}(s)} F_{y}(s) \\
& Y(s)=\frac{G_{y x}(s) \cdot G_{F_{x}}(s)}{1-G_{x y}(s) \cdot G_{y x}(s)} F_{x}(s)+\frac{G_{F_{y}}(s)}{1-G_{x y}(s) \cdot G_{y x}(s)} F_{y}(s)
\end{aligned}
$$

During the experiment of frequency response measurement to gyroscope's drive-mode, $\mathrm{F}_{y}=0$ and $\Omega_{\mathrm{Z}}=0$, thus the transfer function of drive-mode is written as:

$$
G_{X}(s)=\frac{X(s)}{F_{x}(s)}=\frac{G_{F_{x}}(s)}{1-G_{x y}(s) \cdot G_{y x}(s)}=\frac{m s^{2}+D_{22} s+k_{22}}{\left(m s^{2}+D_{11} s+k_{11}\right)\left(m s^{2}+D_{22} s+k_{22}\right)-\left(D_{12} s+k_{12}\right)\left(D_{21} s+k_{21}\right)}(19
$$

During the experiment of frequency response measurement to gyroscope's detect-mode, $\mathrm{F}_{X}=0$ and $\Omega_{z}=0$, thus the transfer function of detect-mode is written as:

$$
G_{Y}(s)=\frac{Y(s)}{F_{y}(s)}=\frac{G_{F_{y}}(s)}{1-G_{x y}(s) \cdot G_{y x}(s)}=\frac{m s^{2}+D_{11} s+k_{11}}{\left(m s^{2}+D_{11} s+k_{11}\right)\left(m s^{2}+D_{22} s+k_{22}\right)-\left(D_{12} s+k_{12}\right)\left(D_{21} s+k_{21}\right)}
$$

Plug the gyroscope parameters into the transfer functions (19) and (20), the frequency response curves are obtained by MATLAB as shown in Fig. 5 .
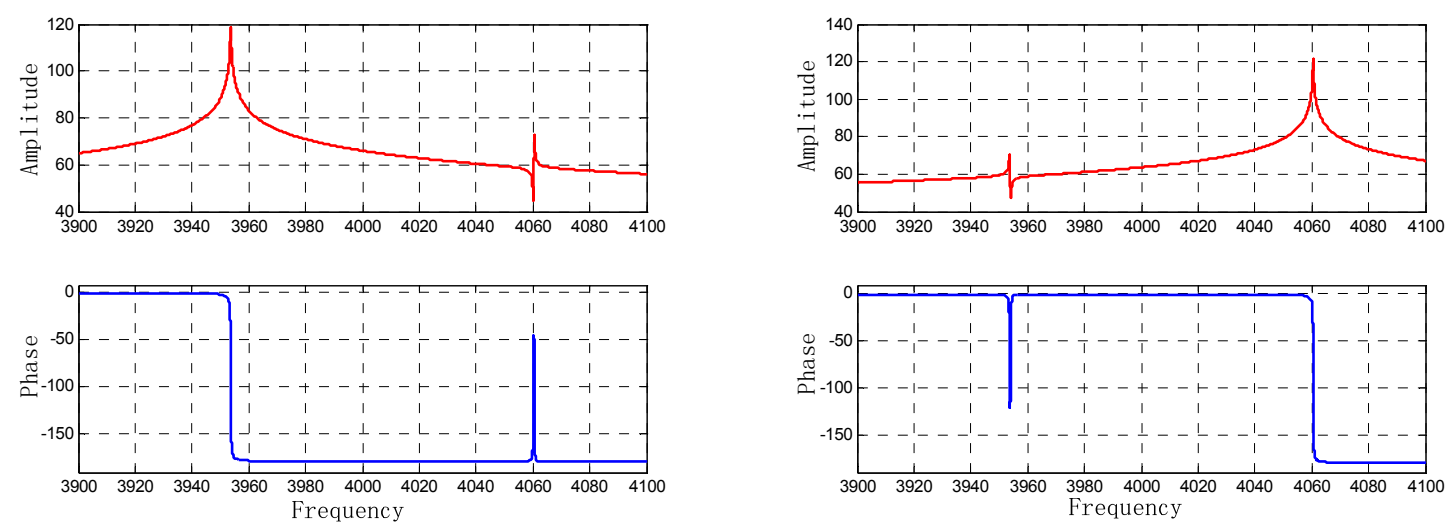

(a) Frequency response curves of $G_{X}(s)$

(b) Frequency response curves of $G_{Y}(s)$

Fig.5 Frequency Response Curves of $G_{X}(s)$ and $G_{Y}(s)$

Compared with Fig.3, the characteristics of the frequency response curves in Fig. 5 fit the results of the frequency response measuring experiment well, which verifies the correctness of the above mathematical model of silicon micro-machined gyroscope mode-coupling system. 


\section{Summary}

Comparing between the idealized frequency response curves with the experiment results of frequency response measurement, the mode-coupling characteristics of silicon micro-machined gyroscope system are discussed in this paper. The non-ideal factors are analyzed from three main perspectives: structural asymmetry, unbalanced driving force, and principium factor. According to the analysis and the non-idealized dynamic model, paper deducted the dynamic equations and transfer functions of mode-coupling gyroscope system, and obtained the mathematical model of silicon micro-machined gyroscope mode-coupling system. Through the simulating experiment by MATLAB, the validity of the mathematical model was discussed, which fit the experimental results well.

\section{Acknowledgements}

Thanks for the support provided by Chongqing Natural Science Foundation (cstc2013jcyjA40035).

\section{References}

[1] K.Najafi, J.Chae, H.Kulah and Guohong He. Micromachined Silicon Accelerometers and Gyroscopes [C]. Proceedings of the 2003 IEEE/RSJ, International Conference on Intelligent Robots and Systems, Las Vegas, Nevada, 2003: 2353-2358.

[2] W.Zhou, B.Zhou, Z.Chen and R.Zhang. Effects of Combs on Coupling Error in the Vibratory MEMS Gyroscope with Various Air Dampings[J]. The Ninth International Conference on Electronic Measurement \& Instruments, 2009: 298-301

[3] Xiao Dingbang. Study on Key Technologies of a Novel Butterfly Micromacined Gyroscope [D]. Changsha, Hunan, China: National University of Defense Technology, 2009

[4] Reiner M.Dreizler, Cora S.Ludde. Theoretical Mechanics, First Edition, 2010. 